\title{
Sur le problème de la mesure.
}

\author{
Par \\ Stefa n B a nach (Léopol = Lwów).
}

\section{Introduction.}

Dans ce travail je m'occupe du probleme de la mesure et des problèmes connnexes qui s'attachent aux questions suivantes:

Dans son livre "Lecons sur l'intégration" (Paris 1905) M. Lebesgue énonce les propriétés de son intégrale:

$1^{0}$. Quels que soient $a, b, h$, on a

$$
\int_{a}^{h} f(x) d x=\int_{a+n}^{b+n} f(x-h) d x .
$$

$2^{0}$. Quels que soient $a, b, c$, on a

$$
\int_{a}^{b} f(x) d x+\int_{0}^{b} f(x) d x+\int_{e}^{a} f(x) d x=0
$$

30. $\quad \int_{a}^{b}[f(x)+\varphi(x)] d x=\int_{a}^{b} f(x) d x+\int_{a}^{b} \varphi(x) d x$.

40. Si l'on a $f \geqslant 0$ et $b>a$, on a aussi $\int_{a}^{b} f(x) d x \geqslant 0$.

50. On a: $\int_{0}^{1} 1 \cdot d x=1$.

60. Si $f_{n}(x)$ tend en croissant vers $f(x)$, l'intégrale de $f_{n}(x)$ tend vers celle de $f(x)$.

En même temps M. Lebesgue pose le problème si la propriété 60 est indépendante de cinq autres, ce qu'il faut comprendre comme le problème si une intégrale jouissant des propriétés $1^{\circ}-5^{0}$ 
pour un ensemble de fonctions, jouit nécessairement de la propriété $6^{\circ}$.

Dans son livre „Grundzüge der Mengenlehre" (Leipzig 1914), p. 469 ss., M. Hausdorff s'occupe du problème suivant, qu'on peut appeler problème large de la mesure: Peut-on attacher à chaque ensemble borné $E$ d'un espace à $m$ dimensions un nombre $m(E)$ satisfaisant aux conditions suivantes:

1) $m(E) \geqslant 0$

2) $m\left(E_{0}\right)=1$ pour un ensemble $E_{0}$ de l'espace considéré,

3) $m\left(E_{1}+E_{2}\right)=m\left(E_{1}\right)+m\left(E_{2}\right)$, si $E_{1} E_{2}=0$,

4) $m\left(E_{1}\right)=m\left(E_{2}\right)$ si les ensembles $E_{1}$ et $E_{2}$ sont superposables ${ }^{1}$ ).

Il prouve que ce problème est imposible pour l'espace à trois ou plus dimensions. Il démontre notamment (à l'aide de l'axiome de M. Zermelo) l'existence d'une décomposition de la surface d'une sphère en quatre ensembles $A, B, C, D$, où $D$ est un ensemble dénombrable et $A \cong B \cong C, A \cong B+C$.

S'il existait une mesure satisfaisant aux conditions 1)-4), l'ensemble $D$ aurait une mesure nulle et les conditions $1,-4$ ) donneraient:

$$
\begin{gathered}
m(A)=m(B)=m(C)=\frac{1}{8}, \\
m(A)=m(B+C)=\frac{1}{2}
\end{gathered}
$$

ce qui implique une contradiction.

Un problème analogue n'est pas encore résolu pour l'espace à une ou deux dimensions.

M. S. Ruziewicz m'a posé le problème suivant:

Existe-il une opération $f(X)$ satisfaisant aux conditions suivantes:

1) $f(X)$ est définie pour tout ensemble mesurable $(L)$ d'un espace à $n$ dimensions.

2) $f(X) \geqslant 0$.

3) $f\left(X_{0}\right)=1$ pour un certain ensemble $X_{0}$ tel que $m\left(X_{0}\right)=1$.

4) $f(X+Y)=f(X)+f(Y)$ pour $X . Y=0$.

5) $f(X)=f(Y)$ si $X \cong Y$.

6) $f\left(X_{1}\right) \neq m\left(X_{1}\right)$ pour un certain ensemble $X_{1}$ mesurable $(L)$.

1) Nous écrirons $E_{1} \cong E_{2}$ pour exprimer que les ensembles $E_{1}$ ot $E_{2}$ sont superposables (par translation on rotation antour d'un centro on autour d'an axe). 
$J_{\Theta}$ démontre dans ce travail qu'on peut attribuer une mesure à tout ensemble linéaire et à tout ensemble dans le plan. Je prouve aussi qu'on peut attacher à toute fonction bornée d'une variable réelle $f(x)$ et à tout intervalle $(a, b)$ un nombre $\int_{a}^{b} f(x) d x$ satisfaisant aux conditions $1^{0}-5^{0}$ de M. Lebesgue: ce nombre peut etre regardé comme une généralisation de l'intégrale. Pour une fonction intégrable au sens de Riemann ce nombre est son intégrale rimanienne; pour les fonctions intégrables au sens de Lebesgue ce nombre ne coïncide pas nécessairement avec son intégrale lebes-

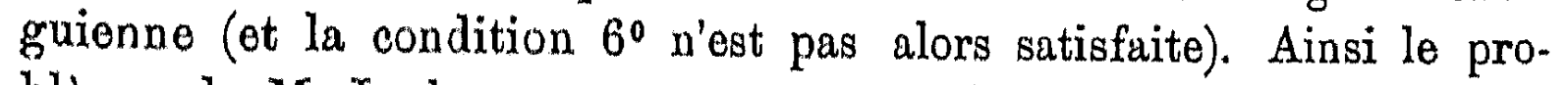
blème de M. Lubesgue se trouve résolu négativement, c.-̀̀-d. la condition $6^{0}$ est indépendante des conditions $1^{0}-5^{0}$. En ce qui concerne le problème de M. Hausdorff, nous voyons qu'il est autrement pour les espaces à une ou deux dimensions, où on peut attribuer une mesure à chaque ensemble, et autrement pour les espaces à $n \geqslant 3$ dimensions, où cela est impossible.

Le problème de M. Ruziewicz se trouve résolu affirmativement pour les espaces à une et deux dimensions, cependant pour les espaces à plusieurs dimensions lo probleme reste ouvert.

Toutes les fonctions considérées dans ce travail seront bornées, c.-à-d. pour toute fonction $f(x)$ existe un nombre $M(f(x))>0$, tel que $|f(x)|<M$ pour tout $x$ pour lequel $f(x)$ est définie.

Soit $f(x)=f(x+1)$ une fonction de période 1, définie sur la circonférence d'un cercle de rayon $=1 / 2 \pi$, ayant pour centre l'origíne des coordonnées rectangulaires; $x$ désigne l'arc correspondant.

Définition 1. Nous appellerons la fonction $f(x)$ équivalente à zéro, en écrivant

$$
f(x) \sim 0
$$

s'il existe pour tout nombre positif $\varepsilon$ une suite finie de nombres réels $\alpha_{1}, \alpha_{2}, \ldots, \alpha_{n}$, telle qu'on a pour tout $x$ réel

$$
\frac{1}{n}\left|\sum_{k=1}^{n} f\left(x+\alpha_{k}\right)\right|<\varepsilon
$$


Théorème 1. Si $f(x) \sim 0$ et $g(x) \sim 0$, on a aussi

$$
f(x)+g(x) \sim 0 .
$$

Démonstration. D'après l'hypothèse il existe pour tout $\varepsilon$ positif donné deux suites finies $\alpha_{1}, \alpha_{2}, \ldots, \alpha_{n}$ et $\beta_{1}, \beta_{2}, \ldots, \beta_{r}$ telles qu'on a pour tout $x$ réel

$$
\frac{1}{n}\left|\sum_{k=1}^{n} f\left(x+\alpha_{k}\right)\right|<\varepsilon \quad \text { et } \quad \frac{1}{r}\left|\sum_{l=1}^{r} g\left(x+\beta_{l}\right)\right|<\varepsilon,
$$

ce qui donne sans peine:

$$
\frac{1}{n r}\left|\sum_{k=1}^{n} \sum_{i=1}^{r}\left[f\left(x+\alpha_{k}+\beta_{\imath}\right)+g\left(x+\alpha_{k}+\beta_{\imath}\right)\right]\right| \leqslant 2 \varepsilon ;
$$

il existe donc pour la somme $f(x)+g(x)$ et pour tout $\varepsilon>0$ une suite finie $a_{i}+\beta_{j}(i=1,2, \ldots, n ; j=1,2, \ldots, r)$ jouissant de la propriété exigée par la définition 1 .

Théorème 2. Si $f(x) \sim 0$ et si $C$ est une constante réelle, on $a \cdot C \cdot f(x) \sim 0$.

La démonstration est évidente.

Théorème 3. Si l'on a $f(x) \geqslant C>0$, on n'a pas $f(x) \sim 0$.

Dém. Quel que soit la suite finie $\left\{\alpha_{i}\right\}$, on a toujours

$$
\left|\frac{1}{n} \sum_{i=1}^{n} f\left(x+\alpha_{i}\right)\right| \geqslant \frac{1}{n} \sum_{i=1}^{n} C=C .
$$

Théorème 4. Si $f(x) \sim 0$, si a est une constante réelle, et si l'on pose $\varphi(x)=f(x+a)$, on a

$$
\varphi(x) \sim 0 .
$$

Dém. Lorsque $a_{i}(i=1,2, \ldots, n)$ est une suite correspondante à la fonction $f(x), \alpha_{t}(i=1,2, \ldots, n)$ sera aussi une suite corres. pondante à la fonction $\varphi(x)$.

Définition 2. Nous écrirons

$$
f(x) \sim g(x)
$$

dans ce et seulement dans ce cas, lorsque

$$
f(x)-g(x) \sim 0 .
$$


Théorème 5. Lorsque

$$
f(x) \sim g(x) \quad \text { et } \quad g(x) \sim h(x)
$$

on a

$$
f(x) \sim h(x) .
$$

Dém. D'après (1) et d'après le théorème 1 , on trouve

donc

$$
[f(x)-g(x)]+[g(x)-h(x)] \sim 0,
$$

$$
f(x)-h(x) \sim 0 \text { et } f(x) \sim h(x), \quad \text { c. q. f. d. }
$$

Definition 3. Nous écrirons

$$
f(x) \subsetneq 0
$$

lorsqu'il existe un $C>0$ et une suite finie des nombres réels $\alpha_{1}, \alpha_{2}, \ldots, \alpha_{n}$, tels qu'on a pour tout $x$ réel l'inégalité

$$
\frac{1}{n} \sum_{n=1}^{\infty} f\left(x+\alpha_{i}\right) \geqslant C
$$

Definition 4. Nous écrirons

lorsque

$$
f(x) \zeta 0
$$

$$
-f(x) \Leftarrow 0 .
$$

Définition 5. Nous écrirons

$$
f(x) \succ g(x) \quad \text { (resp. } f(x) \zeta g(x))
$$

lorsque

$$
f(x)-g(x) \lessdot 0 \quad \text { (resp. } f(x)-g(x) \prec 0) .
$$

Théorème 6. Lorsque $f(x) \succ 0$ et lorsque a est une constante réelle, on a

$$
f(x+a)<0 .
$$

Dém. La suite corespondante à la fonction $\varphi(x)=f(x+a)$ est la même qu'à $f(x)$.

Un théorème analogue subsiste pour $f(x) \prec 0$.

Théorème 7. Si

on $a$

$$
f(x) \succ 0 \text { et } g(x) \& 0
$$

$$
f(x)+g(x) \succ 0 .
$$


La démonstration est analogue à la démonstration du théorème 1. Un théorème analogue subsiste pour la relation ${ }^{-} \zeta$.

Théorème 8. Si l'on a pour tout $x$ réel

on n'a pas

$$
f(x) \geqslant 0
$$

$$
f(x) \succ 0 \text {. }
$$

Dém. Il suffit de remarquer qu'on aura pour toute suite $\alpha_{1}, \alpha_{2}, \ldots, \alpha_{n}$ :

$$
\frac{1}{n} \sum_{k=1}^{n} f\left(x+\alpha_{k}\right) \geqslant 0
$$

et de se rapporter à la définition 4.

Théorème 9 . Les relations

$$
f(x) \leqslant 0, f(x) \sim 0, f(x) \succ 0
$$

s'excluent deux d̀ deux.

Dém. Si $f(x)\} 0$, il existe, d'après la déf. 3 , un $C>0$ et une suite finie $\alpha_{1}, \alpha_{2}, \ldots, \alpha_{n}$ tels que

$$
\text { - } \frac{1}{n} \sum_{i=1}^{n} f\left(x+\alpha_{i}\right) \geqslant C \text {. }
$$

S'il était $f(x) \sim 0$, on aurait, d'après les théorèmes 4 et 1:

$$
\frac{1}{n} \sum_{i=1}^{n} f\left(x+\alpha_{i}\right) \sim 0
$$

or les formules (A) et (B) sont incompatibles, d'aprés te théorème 3. Or, en supposant qu'on a simultanément $f(x) \succ 0$ et $f(x) \zeta 0$, on aurait d'après le théorème ô:

$$
f\left(x+\alpha_{k}\right) \precsim 0 \text { pour } k=1,2, \ldots, n,
$$

donc, d'après le théorème 7:

$$
\frac{1}{n} \sum_{k=1}^{n} f\left(x+\alpha_{k}\right) \prec 0
$$

ce qui est impossible d'aprés (A) et le théorème 8.

Pareillement on démontrerait que les relations $f(x) \leqslant 0$ et $f(x) \sim 0$ sont incompatibles. 
Théorème 10. Si

on $a$

$$
f(x) \nvdash g(x) \text { et } g(x) \succ h(x)
$$

$$
f(x) \succ h(x) \text {. }
$$

Dém. On a d'aprés l'hypothèse et la définition 5:

$$
f(x)-g(x) € 0 \text { et } g(x)-h(x) \succ 0,
$$

donc, d'après le théorème 7:

c'est-à-dire

$$
[f(x)-g(x)]+[g(x)-h(x)]<0,
$$

$$
f(x) \nvdash g(x), \quad \text { c. q. f. d. }
$$

Un théorème analogue subsiste pour la relation $\zeta$.

Théorème 11. Si

on a

$$
f(x) \& 0 \text { et } g(x) \sim 0
$$

$$
f(x)+g(x) \succsim 0 .
$$

En partant des définition 3 et 1 on démontre le théorème 11 d'une façon analogue à la démonstration du théorème 1 .

Théorème 12. Si

on $a$

$$
f(x) \succ g(x), \quad f(x) \sim f_{1}(x), \quad g(x) \sim g_{1}(x),
$$

$$
f_{1}(x) \succ g_{1}(x)
$$
avons

Dém. D'après l'hypothèse et d'après les définitions 5 et 2 nous

$$
f(x)-g(x) \gtrless 0, \quad f_{1}(x)-f(x) \sim 0, \quad g(x)-g_{1}(x) \sim 0,
$$

donc, d'aprés le théorème 11:

$$
f_{1}(x)-g_{1}(x) \succ 0
$$

c'est-à-dire $f_{1}(x) \Leftarrow g_{1}(x)$, c. q. f. d.

Considérons maintenant l'ensemble de toutes les fonctions $f(x)$ définies sur la circonférence d'un cercle de rayon $\frac{1}{2 \pi}$, ayant pour centre l'origine des coordonnées. Chacune de ces fonctions sera supposée bornée (mais les fonctions considérées ne seront pas bornées en leur ensemble). 
Divisons toutes les fonctions considérées en classes, en rangeant dans ane méme classe deux fonctions équivalentes et dans les classes differentes deux fonctions qui ne sont pas équivalentes. Une telle classification est possible, d'après le théorème $\bar{b}$. Les classes de fonctions ainsi obtennes seront appellées hyperfonctions et désignées par des majuscules $F, \Phi, F_{1}$ etc. Il est évident que deux hyperfonctions $F_{1}$ et $F_{q}$ sont identiques ou bien n'ont pas d'éléments communs.

Définition 6. Si $F$ est une hyperfonetion dont un élément est 1. functions $f(x)$ et si $C$ est une constante réelle, C.F désignera Thyperfonetion dont un élément est la fonction C. $f(x)$.

Definition 7. Si $F_{1}$ et $F_{2}$ sont des hyperfonctions contenant resp. les fonctions $f_{1}(x)$ et $f_{2}(x)$,

$$
F=F_{1}+F_{1}
$$

designera liyperfonction qui contient la fonction $f(x)=f_{1}(x)+f_{2}(x)$. On prouve ans peine que les hyperfonctions $C . F$, resp. $F_{1}+F_{2}$ we dependent que des hyperfonctions $F$, resp. $F_{1}$ et $F_{2}$.

Définition 8. $-F$ désignera l'hyperfonction $-1 . F$.

D'après les définitions 6,7 et $8, l^{\prime}$ expression

$$
\sum_{k=1}^{n} C_{k} F_{k}
$$

oú $C_{k}$ sont des constantes réelles et $F_{k}$ des hyperfonctions, a un sens et détermine une hyperfonction.

Définition 9. c étant une constante réelle, nous poserons

$$
F=c
$$

ai la hyperfonction $F$ contient la fonction $f(x)=c$.

Définition 10. Nons écrirons

$$
F_{1}>F_{2}
$$

i tonte fonction $f(x)$ qui appartient à l'hyperfonction $F_{1}-F_{2}$ satisfait a la relation

$$
f(x) \succ 0 \text {. }
$$

Théorème 13. $I$ existe pour toute hyperfonction $F$ deux hyperfonctions $F_{1}=c_{1} F_{2}=c_{2}$, telles que

$$
F_{1}>F>F_{2} \text {. }
$$


Démonatration. érant un nombre positif donné ot $f(x)$,un élément do $F$, désignons par $c_{1}$ un nombre réel, tel que

$$
f(x)<c_{1}-\varepsilon \quad \text { pour tout } x \text { réel }
$$

(la fonction $f(x)$ étant bornée, un tol nombre $c_{1}$ existe): nous aurons $c_{1}-f(x)>\varepsilon>0$, done (Déf. 3): $c_{1}-f(x) \leqslant 0$, ce qui domne d'uprés le théoròme 12 ot la déf. 10: $F_{1}^{\prime}>F^{\prime}$.

De merno pour lo signe $<$.

Un ensemble d'hyperfunctions $\Omega$ sora appelé corps, s'il jouit de la propriété suivanto:

Si les hyperfonetions $l_{1}^{\prime}, l_{x}, \ldots, W_{\mu}^{\prime}$ appartiennent $\{\Omega$ et si $c_{1,}, c_{s}, \ldots, c_{n}$ sont dos constantes réllos, l'hyporfonction

appartiont à $\Omega$.

$$
l^{\prime}=c_{1} f_{1}^{\prime}+c_{n} F_{n}+\ldots+c_{n} F_{n}
$$

Dans les considórations ultérieures soront envisagés les corps $\Omega(R)$ et $\Omega(L) . \quad \Omega(R)$ est doffini comme lo plus petit corp jouissant de la propriété suivante:

Toute fonction bornée $f(x)$, intégrable au sens de Riemann appartient à une hyportonction $k$ contenue dans $\Omega(R)$.

On voit sans peine qu'un tel corps existe et que toute hyperfonetion $R$ de ce corps contiont une fonction intégrable au sens de Riemann.

Pareillement on définit le corps $\Omega(L)$ on remplaçant dans la défintion do $\Omega(R)$ les fonctions intégrables au sens do Riomann par les fonctions intégrables au sens de Lobosgue.

Remarque. Soit $\Omega\left(k^{\prime}\right)$ un corps d'hyperfonetions $F, \Phi$ - une hyperfonction qui n'appartient pas à $\Omega(F)$. Formons lo plus petit corps $\Omega(F, \Phi)$ contenant $\Omega(F)$ et l'hyperfonction $\Phi$ : on voit sans peine que $\Omega(F, \Phi)$ se compose d'hyperfonctions de la forme $F+c \Phi$, où $c$ est uno constante et $k^{\prime}$ est uno hyperfonction faisunt partio de $\Omega(F)$.

On roit sans peine que toute hyperfonction $\$ P$ appartenant a $\Omega(R, \Phi)$ peut etro prósentéo d'uno seulo manière sous la formo

$$
\Phi=I+G \Phi .
$$

En effet, en admettant qu'on a oncore la formule

on aurait

$$
y=I^{\prime \prime}+c^{\prime} \Phi,
$$

$$
\left(c-c^{\prime}\right) \Phi=H^{\prime \prime}-H^{\prime}
$$


S'il était $c^{\prime}=c$, on aurait $F^{\prime}=F$ et les décompositions seraient identiques; or il ne peut être $c^{\prime} \neq c$, puisque on aurait

$$
\Phi=\frac{1}{c-c^{\prime}} F^{\prime}-\frac{1}{c-c^{\prime}} F,
$$

et $\Phi$ appartiendrait à $\Omega(F)$, contrairement à l'hypothèse. On en voit aussi que lorsque les hyperfonctions $\dot{\Psi}_{1}, \Psi_{2}$ et $\Psi_{3}$ appartiennent à $\Omega(F, \Phi)$ et lorsqu'on a

et

$$
\Psi_{3}=a \Psi_{1}+b \Psi_{2} \quad \text { (où } a, b \text { sont des const. réelles) }
$$

on a:

$$
\Psi_{1}=F_{1}+c_{1} \Phi, \quad \Phi_{2}=F_{2}+c_{2} \Phi, \quad \Psi_{3}=F_{3}+c_{3} \Phi,
$$

$$
k_{3}^{\prime}=a F_{1}+b F_{2} \quad \text { et } \quad c_{\mathrm{s}}=a c_{1}+b c_{\mathrm{g}}
$$

En effet:

$$
\Psi_{3}=\left(a F_{1}+b F_{8}\right)+\left(a c_{1}+b c_{2}\right) \Phi=F_{3}+c_{3} \Phi
$$

ce qui donne, d'après l'unicité du développement, les relations (I): Définition 11. Soit $\Omega(F)$ un corps d'hyperfonctions $F$. Considérons une opération $A\left(F^{\prime}\right)$ dont l'argument indépendant est uńe hyperfonction $F$ appartenant à $\Omega$ et l'argument dépendant - un nombre réel. Nous dirons que l'opération $A(F)$ est additive, si les conditions que $F_{1}$ et $F_{2}$ appartiennent à $Q$ et que $c_{1}$ et $c_{2}$ sont des constantes réelles, entraînent:

$$
A\left(c_{1} F_{1}+c_{2} F_{2}\right)=c_{1} A\left(F_{1}\right)+c_{2} A\left(F_{2}\right) .
$$

Définition 12. L'opération $A(F)$ est appelée non négative, si pour tout $F^{\prime} \geqslant 0$ appartenant à $\Omega\left(F^{\prime}\right)$ on a: $A\left(F^{\prime}\right) \geqslant 0$.

Thérerme 14. Soit $\Phi$ une hyperfonction n'appartenant pas à $\Omega(F)$ et telle qu'il existe deux hyperfonctions $F_{1}$ et $F_{2}$ de $\Omega(F)$ satisfaisant à l'inégalité

$$
F_{1}>\Phi>F_{2}
$$

et soit $A(X)$ une opération additive et non négative, définie pour les hyperfonctions $X$ de corps $\Omega\left(F^{\prime}\right)$.

Thè se: Il existe une opération additive, non négative $\bar{A}(X)$, définie dans le corps $\Omega(F, \Phi)$ et telle que

$$
\bar{A}(X)=A(X) \text { lorsque } X \text { appartient d } \Omega(F) \text {. }
$$


Dém. D après l'hypothèse, il existe dans $\Omega(F)$ des hyperfonctions $F_{1}>\Phi$; désignons par $\alpha$ la borne inférieure de tous les nombres $A\left(F_{1}\right)$, où $F_{1}$ est une hyperfonction de $\Omega\left(F^{\prime}\right)$ qui est $>\Phi$; $\boldsymbol{\alpha}$ sera donc un nombre fini, non négatif.

Toute hyperfonction $\Psi$ de $\Omega(F, \Phi)$ est de la forme

$$
\Psi=F+c \Phi
$$

où $F$ appartient à $\Omega\left(F^{\prime}\right)$ et $c$ est un nombre réel, et cette décomposition est unique.

Définissons l'opération $\bar{A}(\Psi)$, en posant

$$
\bar{A}(\Psi)=A(F)+c \alpha .
$$

La décomposition (II) étant unique, l'opération $\overline{A(} \Psi)$ est ainsi définie d'une façon uniroque. Lorsque $\Psi$ appartient à $\Omega\left(F^{\prime}\right)$, on a $\bar{A}(\Psi)=A(\Psi)$, puisque alors $\Psi=F+c \alpha$ et $c=0$, donc $\bar{A}(\Psi)=A(F)=A(\Psi)$.

L'opération $\bar{A}(\Psi)$ est additive, puisque $\Psi_{8}=a \Psi_{1}+b \Psi_{2}$ donṇe (v. les formules I):

done

$$
F_{3}=a F_{1}+b F_{1}, \quad c_{3}=a c_{1}+b c_{2},
$$

$$
\begin{aligned}
& \bar{A}\left(\Psi_{3}\right)=A\left(F_{3}\right)+c_{3} \alpha=A\left(a F_{1}+b F_{2}\right)+\left(a c_{1}+b c_{2}\right) \alpha= \\
& =a\left[A\left(F_{1}\right)+c_{1} \alpha\right]+b\left[A\left(F_{2}\right)+c_{2} \alpha\right]=a \bar{A}\left(\Psi_{1}\right)+b \bar{A}\left(\Psi_{2}\right) .
\end{aligned}
$$

Or l'opération $\bar{A}(\Psi)$ est non négative. Soit en effet

$$
\Phi=F+c \Phi \geqslant 0 \text {. }
$$

Si $c=0, \Psi$ appartient à $\Omega(F)$ et on a $\bar{A}(\Psi)=A(\Psi) \geqslant 0$.

Si $c>0$, nous avons $\Phi \geqslant-\frac{1}{c} F$, donc, pour tout $F_{1}>\Phi$ de $\Omega\left(F^{\prime}\right)$ :

$$
F_{1}>-\frac{1}{c}, \text { done } F_{1}+\frac{1}{c}>0
$$

ce qui donne

$$
A\left(F_{1}+\frac{1}{c} F\right)=A\left(F_{1}\right)+\frac{1}{c} A\left(F^{\prime}\right) \geqslant 0,
$$

donc, d'après la définition du nombre $\alpha$ :

$$
\alpha+\frac{1}{c} A\left(F^{\prime}\right) \geqslant 0
$$

donc, $c$ étant $>0: \bar{A}(\Psi)=A(F)+c \alpha \geqslant 0$. 
Si $c<0$, nous avons $-\frac{1}{c} F \geqslant \Phi$, donc $-\frac{1}{c} F>\Phi$, puisque $\Phi$ n'appartient pas à $Q(F)$. D'après la déf. du nombre $\alpha$ nous avons donc $A\left(-\frac{1}{c} F\right) \geqslant \alpha$, ce qui donne, $c$ étant $\langle 0, \bar{A}(\Psi)=A(F)+c \alpha \geqslant 0$.

L'inégalité $\Psi \geqslant 0$ entraîne done toujours l'inégalité $\bar{A}(\Psi) \geqslant 0$ et le théorème est démontré complétement.

Théorème 15. Soit $\left\{\Omega_{\alpha}\right\}(\alpha<\beta)$ un ensemble bien ordonné dis type $\beta$ des corps non décroissants d'hyperfonctions ${ }^{1}$ ), et soit pour tout $\alpha<\beta$ definie dans le corps $Q_{\alpha}$ une operation additive et non négative $A_{\alpha}(X)$, telle que $A_{\alpha}(X)=A_{\xi}(X)$ pour tous le $\xi<\alpha$ et toutes les hyperfonctions $X$ appartenunt $\grave{a} \Omega_{\xi}$. Si l'on désigne $\Omega=\underset{\alpha<\beta}{\sum} \Omega_{\alpha}$ et si l'on pose pour tout $X$ de $Q: \dot{A}(X)=A_{\alpha(X)}(X)$, où $\alpha(X)$ désigne le plus petit nombre ordinal, tel que $X$ appartient à $\Omega_{\alpha(X)}, A(X)$ est une opération additive et non négative, définie dans le corps $\Omega$.

Dém. On voit sans peine qu'une somme (d'un nombre fini ou d'une infinité quelconque) des corps non décroissants d'hyperfonctions est un corps: done $Q=\underset{\alpha<\beta}{\sum} Q_{\alpha}$ est un corps.

Soient $X_{1}$ et $X_{2}$ deux hyperfonctions appartenant à $\Omega, a$ et $b$ deux nombres réels. Soit $\alpha_{0}$ celui de deux nombres $\alpha\left(X_{1}\right)$ et $\alpha\left(X_{\mathbf{2}}\right)$. qui est plus grand (ou leur valeur commune, s'ils sont égaux): nous aurons évidemment $\alpha_{0}<\beta$. Les hyperfonctions $X_{1}$ et $X_{2}$ appartiennent donc toutes les deux à $\Omega_{\alpha_{0}}:$ par conséquent, $A_{\alpha_{0}}(X)$ étant une opération additive dans $\Omega_{\alpha_{0}}$ :

$$
A_{\alpha_{0}}\left(a X_{1}+b X_{2}\right)=a A_{\alpha_{0}}\left(X_{1}\right)+b A_{\alpha_{0}}\left(X_{3}\right) .
$$

Or, d'après l'hypothèse, et d'après $\alpha\left(X_{1}\right) \leqslant \alpha_{0}$ et $\alpha\left(X_{\mathbf{2}}\right) \leqslant \alpha_{0}$ :

$$
A_{\alpha_{\left(X_{1}\right)}}\left(X_{1}\right)=A_{\alpha_{0}}\left(X_{1}\right), \quad A_{\left(X_{2}\right)}\left(X_{2}\right)=A_{\alpha_{0}}\left(X_{2}\right) .
$$

Or, d'après la définition du nombre $a(X), Q_{\alpha_{0}}$ étant un corps contenant $X_{1}$ et $X_{2}$, done aussi $X_{0}=a X_{1}+b X_{2}$ :

done

$$
\alpha\left(X_{0}\right) \leqslant \alpha_{0}
$$

$$
A_{\alpha\left(X_{0}\right)}\left(X_{0}\right)=A_{\alpha_{0}}\left(X_{0}\right)
$$

1) Ceett-d-dire $\Omega_{\xi} \subset Q_{\eta}$ pour $\xi<\eta<\beta$. 
La formule (III) donne done

$$
A_{\alpha\left(X_{0}\right)}\left(X_{0}\right)=a A_{\alpha\left(X_{1}\right)}\left(X_{1}\right)+b A_{\alpha\left(X_{2}\right)}\left(X_{2}\right)
$$

donc, d'après la définition de l'opération $A(X)$ et d'après $X_{0}=$ $=a X_{1}+b X_{2}$ :

$$
A\left(a X_{1}+b X_{2}\right)=a A\left(X_{1}\right)+b A\left(X_{2}\right)
$$

ce qui prouve que l'opération $A(X)$ eșt additive.

Or, il résulte immédiatement de la formule $A(X)=A_{\alpha(X)}(X)$ et de l'hypothèse sur les opérations $A_{\alpha}(X)(\alpha<\beta)$, que l'opération $A(X)$ est non négative.

Notré théorème est ainsi démontré.

Theorème 16. Si $\Omega(F)$ est un corps d'hyperfonctions contenant l'hyperfonction $F=1$ et s'il existe une opération additive et non négative, définie dans $\Omega(F)$, il existe une opération additive et non négative $\bar{A}(X)$, definie pour toute hyperfonction, et telle que $\bar{A}(X)=A(X)$, lorsque $X$ appartient $a \Omega(F)$.

Dém. Soit $\left\{\Phi_{\alpha}\right\}(\alpha<\gamma)$ un ensemble bien ordonné, du type $\gamma$, formé de toutes les hyperfonctions ne faisant pas partie de $\boldsymbol{\Omega}(F)$. Désignons, pour tout $\alpha \leqslant \gamma$, par $\Omega_{\alpha}$ le plus petit corps contenant le corps $\Omega_{0}=\Omega\left(F^{\prime}\right)$ et toutes les hyperfonctions $\Phi_{\xi}$ pour $\xi<\alpha$. (On voit sans peine que, $\mathcal{E}$ désignant un ensemble quelconque d'hyperfonetions, il existe toujours un plus petit corps d'hyperfonction contenant $\mathcal{E}) . \quad\left\{\Omega_{\nu}\right\}(0 \leqslant \alpha \leqslant \gamma)$ sera donc un ensemble bien ordonné non décroissant de corps d'hyperfonction et $\Omega_{\gamma}$ sera le corps de toutes les hyperfonctions.

$\mathrm{J}_{\theta}$ dis qu'on peut définir, par l'induction transfinie, pour tout $\alpha \leqslant \gamma$, une opération additive et non négative $A_{\alpha}(X)$ dans le corps $\Omega_{\alpha}$, telle que $A_{\alpha}(X)=A_{\xi}(X)$ pour tous les $\xi<\alpha$ 'et toutes les hyperfonctions $X$ appartenant à $\Omega_{\xi}$.

En effet, posons $A_{0}(X)=A(X)$ dans $\Omega_{0}=\Omega(F)$.

Soit maintenant $\lambda \geqslant 1$ un nombre ordinal $\leqslant \gamma$ et supposons que pour $0 \leqslant \alpha<\lambda$ sont définies les opérations $A_{\alpha}(X)$ telles que $A_{\alpha}(X)$ est une opération additive et non négative dans $\Omega_{\alpha}$ et que $A_{\alpha}(X)=A_{\xi}(X)$ pour $\xi<\alpha$ et pour les $X$ de $\Omega_{\xi}$. Distinguons doux cas:

1) $\lambda$ est un nombre de première espèce, c'est-à-dire il existe le nombre $\lambda-1 \geqslant 0$. Soit $\Omega_{\lambda-1}=\Omega(\Psi)$ : il résulte de la définition des corps $\Omega_{\alpha}$ qu'on a $\Omega_{\lambda}=\Omega\left(\Psi, \Phi_{\lambda-1}\right)$. Si $\Phi_{\lambda-1}$ appartient a $\Omega_{\lambda-1}$, 
on a $\Omega_{\lambda}=\Omega_{\lambda-1}$ et on peut poser $A_{\lambda}(X)=A_{\lambda-1}(X)$ dans $\Omega_{\lambda}$. Supposons done que $\Phi_{\lambda 1}$ n'appartient pas à $\Omega_{\lambda-1}$. Pour définir l'opération $A_{\lambda}(X)$ nous appliquerons le théorème 14. D'après l'hypothèse, le corps $\Omega_{0}$, donc aussi le corps $\Omega_{\lambda-1}$, contient l'hyperfonction $F=1$, donc toute hyperfonction $F=c, c$ étant un nombre réel quelconque. D'après le théorème 13 , les conditions du théorème 14 sont donc réalisées pour le corps $\Omega_{2-1}$. Donc, d'après le th. 14, on peut définir une opération additive et non négative $A_{\lambda}(X)$ dans le corps $\Omega_{\lambda}=\Omega\left(\Psi, \Phi_{\lambda-1}\right)$, telle que $A_{\lambda}(X)=A_{\lambda-1}(X)$ lorsque $X$ appartient à $\Omega_{\lambda-1}$. D'après la propriété de $A_{\lambda-1}(X)$ nous aurons évidemment aussi $A_{\lambda}(X)=A_{\xi}(X)$ pour $\xi<\lambda$ et pour les hyperfonctions $X$ de $\Omega_{\text {s. }}$.

2) $\lambda$ est un nombre de seconde espèce. Comme on voit sans peine, on a alors $\Omega_{\lambda}=\underset{\alpha \triangleleft \lambda}{\Sigma} \Omega_{\alpha}$, et pour avoir dans $\Omega_{\lambda}$ l'opération $A_{2}(X)$ jouissant des propriétés désirées, on n'a qu'à appliquer le théorème 15.

Nous avons ainsi défini par l'induction transfinie les opérations $A_{\lambda}(X)$ pour $\lambda \leqslant \gamma$ et, en particulier, l'opération $A_{\gamma}(X)$. L'opération $A_{\gamma}(X)$, définie dans le corps $\Omega_{\gamma}$ de toutes les hyperfonction, jouit évidemment des propriétés démandées par le théorème 16.

Le théorème 16 est ainsi démontré.

\section{Applications.}

Théorème 17. Toute fonction $f(x)$ intégrable au sens de Rie$\operatorname{man}{ }^{1)}$ satisfait à la relation

$$
f(x) \sim c, \text { où } \quad c=\int_{0}^{1} f(x) d x .
$$

Dém. Comme on voit sans peine, l'expression

$$
\frac{1}{n} \sum_{k=1}^{n} f\left(x+\frac{k}{n}\right)
$$

converge (pour une fonction intégrable $R$ ) uniformément vers $\int_{0}^{1} f(x) d x$ pour $n=\infty$, donc

1) Rappelons qu'il s'agit toujours des fonctions bornées, deffinies sur une circonférence. 


$$
\left|\left[\frac{1}{n} \sum_{k=1}^{n} f\left(x+\frac{k}{n}\right)\right]-c\right|<\varepsilon \text { pour } n>\mu(\varepsilon),
$$

c'est-à-dire

$$
\left|\frac{1}{n} \sum_{k=1}^{n}\left[f\left(x+\frac{k}{n}\right)-c\right]\right|<\varepsilon \quad \text { pour } \quad n>\mu(\varepsilon),
$$

ce qui démontre que $f(x)-c \sim 0$, donc $f(x) \sim c$, c. q. f. d.

Théorème 18. Il existe une fonction bornée $\varrho(x)$, dont l'intégrale lebesguienne est nulle et telle que toute fonction $\varphi(x)$ intégrable au sens de Riemann et satisfaisant $\grave{a}$ la relation

$$
\varphi(x) \succ \rho(x),
$$

vérifie l'inégalite

$$
\int_{0}^{1} \varphi(x) d x>1
$$

Dé m. Considérons sur la circonférence un ensemble $E$ de mesure lebesguienne nulle, dont le complémentaire est de première catégorie de M. Baire. La fonction $\varrho(x)$ égale à 1 sur $E$ et à 0 sur le complémentaire de $E$ satisfait aux conditions de notre théorème.

En effet, il est clair que

$$
(L) \int_{0}^{1} \varrho(x) d x=0 .
$$

Or, soit $\varphi(x)$ une fonction intégrable $(R)$ et satisfaisant à la relation (1). Il existe done une suite finie $\alpha_{1}, \alpha_{i}, \ldots \alpha_{n}$ et un nombre $\varepsilon>0$, tels que

$$
\frac{1}{n} \sum_{i=1}^{n}\left[\varphi\left(x+\alpha_{i}\right)-\varrho\left(x+\alpha_{t}\right)\right]>\varepsilon
$$

pour tout $\boldsymbol{x}$ réel. Il en résulte tout de suite que pour tout $k$ naturel et tout $x$ réel subsiste l'inégalité

done

$$
\frac{1}{k} \sum_{j=1}^{k}\left\{\frac{1}{n} \sum_{i=1}^{n}\left[\varphi\left(x+\alpha_{i}+\frac{j}{k}\right)-\rho\left(x+\alpha_{i}+\frac{j}{k}\right)\right]\right\}>\varepsilon
$$

$$
\frac{1}{n} \sum_{i=1}^{n}\left\{\frac{1}{k} \sum_{j=1}^{k} \varphi\left(x+\alpha_{i}+\frac{j}{k}\right)\right\}-\frac{1}{n} \sum_{i=1}^{n}\left\{\frac{1}{k} \sum_{j=1}^{k} \varrho\left(x+\alpha_{i}+\frac{j}{k}\right)\right\}>\varepsilon .
$$


Pour $k>\mu$ (et tout $x$ réel) la première de ces sommes diffère de $\int_{0}^{1} \varphi(x) d x$ à moins que de $\varepsilon$. Or, je dis qu'il existe des nombres $x$ pour lesquels la seconde des sommes $(3)$ est $=1$. Désignons, en effet; par $E\left(\alpha_{i}+\frac{j}{k}\right)$ l'ensemble qu'on obtient en tournant l'ensemble $E$ (autour du centre de la circonférence considérée) d'un angle $\alpha_{i}+\frac{j}{k}$. L'ensemble $E$ ayant pour complémentaire un ensemble de première catégorie, il en est de même pour tout ensemble $E\left(a_{i}+\frac{j}{k}\right)$, pour $i=1,2, \ldots n ; j=1,2, \ldots k$ : leur produit $\Pi$ est donc un ensemble de deuxième catégorie. Pour tout nombre $x$ de $I$ nous aurons évidemment

$$
\frac{1}{n} \sum_{i=1}^{n}\left\{\frac{1}{k} \sum_{j=1}^{k} \varrho\left(x+\alpha_{i}+\frac{j}{k}\right)\right\}=1
$$

donc, linégalité (3) subsistant pour tous les $x$ réels:

$$
\int_{0}^{1} \varphi(x) d x+\varepsilon-1>\varepsilon
$$

ce qui donne l'inégalité (2).

Remarque 1. La borne inférieure de toutes les intégrales de toutes les fonctions intégrables $R$ et satiafaisant à la relation (1) est $=1$, puisque pour tout $\varepsilon>0$ la fonction $\varphi(x)=1+\varepsilon$ vérifié cette relation.

Remarque 2. La fonction $\rho(x)$ n'est équivalente à aucane fonction $\varrho(x)$ intégrable $R$.

En effet, s'il était

$$
\rho(x) \sim \varphi(x), \text { où } \varphi(x) \text { est intégrable } R,
$$

alors, on désignant $\int_{0}^{1} \varphi(x) d x=c$, on aurait, d'après le théorèmè 17:

$$
\rho(x) \sim c, \quad \rho(x)-c \sim 0,
$$


donc, pour tout $\varepsilon>0$ donné et pour une suite $a_{1}, \alpha_{2}, \ldots, \alpha_{n}$ convenable:

$$
\left|\frac{1}{n} \sum_{n=1}^{n}\left[\rho\left(x+\alpha_{k}\right)-c\right]\right|<\varepsilon, \text { pour tout } x .
$$

Or, il résulte sans peine de la définition de la fonction $\rho(x)$ qu'il existe des points $x$ dans lesquels l'expression $\frac{1}{n} \sum_{k=1}^{n} \rho\left(x+\alpha_{k}\right)$ prend la valeurs 1 , et des points $x$ (formant un ensemble de mesure 1) dans lesquels cette expression prend la valeur 0 . L'inégalité (4) est donc impossible.

Theorème 19. Il existe une opération $H$, dont le contre-domaine est ensemble des nombres réels, définie pour toute fonction bornée (définie sur la circonférence), satisfaisant aux conditions suivantes:

1) Si $\dot{f}_{1}(x)$ et $f_{\mathbf{z}}(x)$ sont deux fonetions (bornées), $c_{1}$ et $c_{2}$ deux nombres réels, on a

$$
H\left(c_{1} f_{1}(x)+c_{1} f_{2}(x)\right)=c_{1} H\left(f_{1}(x)\right)+c_{2} H\left(f_{2}(x)\right) .
$$

2) Si $f_{1}(x) \geqslant 0$ pour tout $x$ (sur la circonférence), on a

$$
H\left(f_{1}(x)\right) \geqslant 0 \text {. }
$$

3) Si $f_{1}(x)=c$, on a $H\left(f_{1}(x)\right)=c$.

4) Pour tout a réel on $a$

$$
H(f(x))=H(f( \pm x+\alpha))
$$

5) Lorsque $f(x)$ est intégrable $(L)$, on a

$$
H(f(x))=(L) \int_{0}^{1} f(x) d x
$$

D ém. Considérons le corps $\Omega(L)$. En conservant les notations du théorème 16, posons pour les hyperfonctions $X$ de $\Omega(L)$ :

$$
A(X)=(L) \int_{0}^{1} \varphi(x) d x
$$

où $\varphi(x)$ est ure fonction intégrable $(L)$ faisant partie de l'hyperfonction $X$. 
Pour prouver que $A(X)$ est bien déterminé pour tout $X$ de $\boldsymbol{\Omega}(L)$, il suffira évidemment de démontrer qu'on a

$$
\text { (L) } \int_{0}^{1} f(x) d x=0
$$

pour toute fonction $f(x)$ intégrable $(L)$ et satisfaisant à la relation

( $\beta$ )

$$
f(x) \sim 0 .
$$

D'après ( $\beta$ ) il existe pour tout $\varepsilon>0$ une suite $\alpha_{1}, \alpha_{2}, \ldots, \alpha_{n}$ telle que

$$
\left|\frac{1}{n} \sum_{k=1}^{n} f\left(x+\alpha_{k}\right)\right|<\varepsilon, \text { donc }(L) \int_{0}^{1}\left|\frac{1}{n} \sum_{k=1}^{n} f\left(x+\alpha_{k}\right)\right| d x<\varepsilon,
$$

et, a plus forte raison:

$(\gamma)$

$$
\left|(L) \int_{0}^{1} \frac{1}{n} \sum_{k=1}^{n} f\left(x+\alpha_{k}\right) d x\right|<\varepsilon
$$

or:

$$
\text { (L) } \int_{0}^{1} f(x) d x=(L) \int_{0}^{1} f\left(x+\alpha_{k}\right) d x=\frac{1}{n} \sum_{k=1}^{n}(L) \int_{0}^{1} f\left(x+\alpha_{k}\right) d x
$$

(puisque $f(x+1)=f(x)$ ): donc, d'après $(\gamma)$ :

(

$$
\left|(L) \int_{0}^{1} f(x) d x\right|<\varepsilon
$$

le nombre $\varepsilon$ étant aussi petit que l'on veut, l'inégalité $(\delta)$ donne l'égalité ( $\alpha$ ), c. q. f. d.

Si $X \geqslant 0$, alors, en désignant par $\varphi(x)$ une fonction quelconque intégrable $(L)$ et faisant partie de $X$, nous aurons

$$
\text { soit } \varphi(x) \& 0, \text { soit } \varphi(x) \sim 0 \text {. }
$$

Dans le premier cas nous avons pour une certaine suite $a_{1}, \alpha_{2}, \ldots, \alpha_{n}$ et certain $c$ :

$$
\frac{1}{n} \sum_{k=1}^{n} \varphi\left(x+\alpha_{k}\right)>c, \text { donc } \frac{1}{n} \sum_{k=1}^{n}(L) \int_{0}^{1} \varphi\left(x+\alpha_{k}\right) d x>c,
$$

c'est-à-dire

$$
(L) \int_{0}^{1} \varphi(x) d x>c
$$


donc

$$
A(X)>c>0 .
$$

Or, nous avons démontré que pour les fonctions intégrables $(L)$ la relation $(\beta)$ entraîne l'inégalité $(\alpha)$. Il en résulte tout de suite que dans le second cas $(\varrho(x) \sim 0)$ nous aurons $A(X)=0$.

Nous avons done pour tout $X \geqslant 0$ de $\Omega(L)$ :

$$
A(X) \geqslant 0 \text {. }
$$

L'opération $A(X)$ est donc non négative.

Or, l'opération $A(X)$ est additive: en effet, si

$X=c_{1} X_{1}+c_{2} X_{2}, \quad$ (où $X, X_{1}$ et $X_{2}$ appartiennent à $\Omega(L)$ ),

alors, en désignant resp. par $\varphi_{1}(x), \varphi_{\mathbf{2}}(x)$ des fonctions intégrables $(L)$ et faisant partie de $X_{1}, X_{2}$, nous aurons:

$$
\varphi(x)=c_{1} \varphi_{1}(x)+c_{2} \varphi_{2}(x)
$$

où $\varphi(x)$ appartient à $X$, ce qui donne:

$$
\begin{gathered}
A(X)=(L) \int_{0}^{1}\left(c_{1} \varphi_{1}(x)+c_{2} \varphi_{2}(x)\right) d x= \\
=c_{1}(L) \int_{0}^{1} \varphi_{1}(x) d x+c_{2}(L) \int_{0}^{1} \varphi_{2}(x) d x=c_{1} A\left(X_{1}\right)+c_{2} A\left(X_{2}\right) .
\end{gathered}
$$

Or $\Omega(L)$ contient l'hyperfonction $X_{0}=1$. Les conditions du théorème 16 sont donc réalisées. En vertu de ce théorème il existe donc une opération additive et non négative $\bar{A}(X)$, défnie pour toute hyperfonction $X$ et telle que $\overline{A(X)}=A(X)$ pour tout $X$ de $\Omega(L)$.

Définissons maintenant une opération $G(f(x))$, dont le domaine est l'ensemble de toutes les fonctions (bornées à période 1) et le contre-domaine - l'ensemble de nombres réels $(\geqslant 0)$, comme il suit: Si $f(x)$ appartient à l'hyperfonction $X$, posons

$$
G(f(x))=\bar{A}(X) .
$$

L'opération $G$ est déterminée d'une façon univoque puisque $f(x)$ appartient à un seul $X$. Elle est additive, puisque si

$$
f(x)=c_{1} f_{1}(x)+c_{2} f_{2}(x),
$$


les hyperfonctions correspondantes satisfont à la relation

$$
X=c_{1} X_{1}+c_{3} X_{2},
$$

ce qui donne, $\bar{A}(X)$ étant une operation additive:

$$
\bar{A}(X)=c_{1} \bar{A}\left(X_{1}\right)+c_{2} \bar{A}\left(X_{2}\right),
$$

done .

$$
G(f(x))=c_{1} G\left(f_{1}(x)\right)+c_{2} G\left(f_{\mathrm{g}}(x)\right)
$$

Or, $G(f(x)) \geqslant 0$ pour $f(x) \geqslant 0$. En effet, envisageons la fonction

$$
\varphi(x)=c+f(x), \text { où } c>0 \text { : }
$$

nous aurons $\varphi(x) \geqslant c$ (pour tout $x$ réel), donc

$$
\varphi(x) \succeq 0
$$

l'hyperfonction correspondante $\Phi$ vérifie donc l'inégalité $\Phi>0$, donc $A(\Phi) \geqslant 0$, ce qui donne $G(\varphi(x)) \geqslant 0$, donc

$$
G(c+f(x)) \geqslant 0,
$$

ce qui donne ( $G$ étant une opération additive):

$$
G(f(x)) \geqslant-G(c)=-c,
$$

donc, $c$ étant un nombre positif quelconque:

$$
G(f(x)) \geqslant 0, \quad \text { c. q. f. d. }
$$

L'opération $G$ vérifie aussi l'égalité

$$
G(f(x+\alpha))=G(f(x)),
$$

puisque nous avons $f(x+\alpha) \sim f(x)$ et parsuite les deux fonctions appartiennent à la même hyperfonction $X$.

Or, il est elair que

$$
G(f(x))=(L) \int_{0}^{1} f(x) d x
$$

lorsque $f(x)$ est intégrable $(L)$.

Définissons maintenant l'opération $H(f(x))$ en posant

$$
H(f(x))=\frac{1}{2} G(f(x))+\frac{1}{2} G(f(-x)) .
$$

Cette opération vérifie les conditions 1)-5) de notre théorème. 
1) Elle est additive, puisque $G$ l'est.

2) Pour $f(x) \geqslant 0$ nous avons $f(-x) \geqslant 0$, donc $G(f(x)) \geqslant 0$; $G(f(-x)) \geqslant 0$ et $H(f(x)) \geqslant 0$.

3) Pour $f(x)=c$ nous avons

$$
H f(x))=\frac{1}{2} G(c)+\frac{1}{2} G(c)=G(c)=c .
$$

4) Nous avons

$$
\begin{gathered}
H(f( \pm x+\alpha))=\frac{1}{2} G(f( \pm x+\alpha))+\frac{1}{2} G(f(\mp x-\alpha))= \\
=\frac{1}{2} G(f( \pm x))+\frac{1}{2} G(f(\mp x))=H(f(x)) .
\end{gathered}
$$

5) Lorsque $f(x)$ est intégrable $(L)$, nous avons, d'après $f(x)=$ $=f(x+1)$ :

$$
\begin{gathered}
H(f(x))=\frac{1}{2} G(f(x))+\frac{1}{2} G(f(-x))=\frac{1}{2} G(f(x))+\frac{1}{2} G(f(1-x))= \\
=\frac{1}{2}(L) \int_{0}^{1} f(x) d x+\frac{1}{2}(L) \int_{0}^{1} f(1-x) d x=(L) \int_{0}^{1} f(x) d x .
\end{gathered}
$$

Le théorème 19 est ainsi démontré complètement.

Thérème 20. Il existe une opération $H$ vérifiant les conditions 1)-4) du theorème 19 qui n'est pas identique à l'intégrale de Lobesgue pour les fonctions bornées intégrables $(L)$.

Dém. Considérons le corps $\Omega(R)$ et désignons par $P$ l'hyperfonction qui contient la fonction $\rho(x)$ définie dans le théorème 18 (qui, comme nous avons observé dans la remarque 2 au th. 18, n'appartient à aucune hyperfonction de $\Omega(R)$ ).

Posons dans le corps $\Omega(R)$ :

$$
A(X)=\int_{0}^{1} \varphi(x) d x,
$$

où $\varphi(x)$. est une fonction intégrable $R$, contenue dans $X$. Pour définir dans le corps $\Omega_{1}=\boldsymbol{Q}(R, P)$ l'opération additive et non négative $A_{1}(X)$, telle que $A_{1}(X)=A(X)$ dans $\Omega(R)$, nous pouvons poser

$$
A_{1}(R+c P)=A(R)+c
$$

(voir la dém. du th. 14), puisque, d'après la remarque $I$ au théorème 18 et d'après $(\varepsilon)$, la borne inférieure $\alpha$ de tous les nombres $A(X)$ pour les hyperfonctions $X$ de $\Omega(R)$ vérifiant l'inégalité $X>P$ est $=1$. 
De $(\eta)$ résulte en particulier que

$$
A_{1}(P)=1 \text {. }
$$

En appliquant maintenant le théorème 16, nous concluons qu'il existe une opération additive et non négative $\bar{A}(X)$ définie pour toute hyperfonction et telle que $\overline{A(X)}=A_{1}(X)$ pour les $X$ de $\Omega_{1}=\Omega(R, P)$, donc

(2).

$$
\bar{A}(P)=1 \text {. }
$$

En définissant maintenant les opérations $G(f(x))$ et ensuite $H(f(x))$ comme plus haut, nous vérifions sans peine que $H$ jouit des propriétés 1) - 4) du théorème 19 et que (d'après $(\zeta), \varrho(x)$ appartenant à $P$ ):

$(\mu)$

$$
H(\rho(x))=1
$$

tandis que

$$
\text { (L) } \int_{0}^{1} \rho(x) d x=0
$$

(voir la dém. du th. 18). Le théorème 20 est ainsi établi.

Remarque I. On peut démontrer sans peine que pour les opérations $H(f(x))$ vérifiant les conditions 1) -4) du théorème 19 subsiste. la formule

$$
H(f(x))=\int_{0}^{1} f(x) d x
$$

lorsque $f(x)$ est intégrable $R$.

Observons à ce but d'abord que

$$
H(f(x))=H(g(x)), \text { si } f(x) \sim g(x) .
$$

Il suffira évidemment démontrer qu'on a

$$
H(\varphi(x))=0, \text { si } \varphi(x) \sim 0
$$

et appliquer ensuite la propriété 1) de l'opération $H$.

Soit donc $\varphi(x) \sim 0$. Il existe donc pour tout $\varepsilon>0$ une suite finie $a_{1}, a_{2}, \ldots, a_{n}$ telle qu'on a pour tout $x$ l'inégalité

$$
-\varepsilon \leqslant \frac{1}{n} \sum_{n=1}^{n} \rho\left(x+\alpha_{k}\right) \leqslant \varepsilon .
$$


Or, d'après les propriétés 1) et 4) de l'opération $H$ :

$$
H\left[\frac{1}{n} \sum_{k=1}^{n} \varphi\left(x+\alpha_{k}\right)\right]=\frac{1}{n} \sum_{k=1}^{n}\left(\varphi\left(x+\alpha_{k}\right)\right)=H(\varphi(x))
$$

l'inégalité (iii) donne donc

$$
-\varepsilon \leqslant H(\varphi(x)) \leqslant \varepsilon .
$$

Le nombre positif $\varepsilon$ étant quelconque, cela prouve la formule (ii). $\mathrm{La}$ formule (i) est ainsi établie.

Or, lorsque $f(x)$ est intégrable $R$, on a (th. 17):

$$
f(x)=c=\int_{0}^{1} f(x) d x
$$

done, d'après la propriété 3) de l'opération $H$ :

$$
H(f(x))=H(c)=e=\int_{0}^{1} f(x) d x, \quad \text { c. q. f. d. }
$$

Remarque II. En posant

$$
\int_{a}^{b} f(x) d x= \begin{cases}H(\varphi(x)) & \text { pour } \quad b \geqslant a \\ -H(\varphi(x)) & \text { pour } \quad b<a,\end{cases}
$$

où

$$
\varphi(x)=\left\{\begin{array}{c}
f(x) \text { dans lintervalle }(a, b) \\
0 \text { pour les autres } x,
\end{array}\right.
$$

et où $H$ est une opération satisfaisant aux conditions du théorème 20 , nous obtenons une intégrale satisfaisant aux conditions $1^{\circ}-5^{\circ}$ de M. Lebesgue énumérées dans l'Introduction, mais ne satisfaisant pas à la condition $6^{\circ}$.

En effet, d'après M. Lebesgue, les conditions $1^{0}-6^{0}$ caracté-. risent son intégrale, donc, l'intégrale que nous venons de définir satisfaisant aux conditions $1^{\circ}-5^{0}$ et ne coincidant pas (pour les fonctions intégrables $L$ ) avec celle de $\mathbf{M}$. Lebesgue (d'après $(\mu$.) et (v)), elle ne peut pas satisfaire à la condition $6^{\circ}$.

Or, on pourrait sans peine démontrer directement que notre intégrale ne satisfait pas à la condition $6^{\circ}$ pour les fonctions intégrables $(L)$. En effet, la fonction $\varrho(x)$ étant nulle sauf dans un ensemble de mesure nulle, il existe une suite infinie d'intervalles $\delta_{1}, \delta_{2}, \delta_{3}, \ldots$ recourrant $E$, dont la somme des longueurs est $\varepsilon<1$. 
Soit $f(x)$ une fonction égale à 1 pour les $x$ appartenant à un $\delta_{i}$ et a 0 pour les autres $x$. Nous aurons évidemment pour tout $x$

donc

$$
\rho(x) \leqslant f(x) \leqslant 1
$$

et

$$
H(\rho(x)) \leqslant H(f(x)) \leqslant H(1)=1,
$$

$$
H(f(x))=1 \quad \text { (puisque } H(\varrho(x))=1) .
$$

Or, posons

$$
f_{n}(x)=\left\{\begin{array}{l}
1 \text { lorsque } x \text { appartient à } \delta_{1}+\delta_{2}+\ldots+\delta_{n} \\
0 \text { pour les autres } x
\end{array}\right.
$$

nous aurons

$$
f_{1}(x) \leqslant f_{2}(x) \leqslant f_{3}(x) \leqslant \cdots
$$

et la suite $f_{n}(x)$ converge évidemment (non uniformément) vers $f(x)$. Cependant

$$
\lim _{n \rightarrow \infty} H\left(f_{n}(x)\right) \leqslant \varepsilon<1=H(f(x)) .
$$

Le théorèmes démontrés nous permettent d'énoncer les propositions suivantes:

Théorème I. On peut attacher à tout ensemble $E$ de points d'une circonférence de rayon $\frac{1}{2 \pi}$ un nombre $H(E)$ satisfaisant aux conditions suivantes:

1) $H(E) \geqslant 0$.

2) $H\left(E_{0}\right)=1$ lorsque $E_{0}$ désigne l'ensemble de tous les points de la circonférence.

3) $H\left(E_{1}+E_{2}\right)=H\left(E_{1}\right)+H\left(E_{2}\right)$, si $E_{1} E_{\mathrm{q}}=0$.

4) $H\left(E_{1}\right)=H\left(E_{2}\right)$, si $E_{1} \cong E_{2}$.

5) $H(E)$ coïncide avec la mesure lebesguienne de l'ensemble $E$ lorsque $E^{2}$ est mesurable $(L)$.

Théorème II. Il existe une fonction $H(E)$ vérifiant les conditions 1)-4) du théorème $I$ et un ensemble $W$ de mesure lebesguienne nulle, tels que $H(W)=1$.

Pour démontrer ces théorèmes, il suffit de poser

$$
H(E)=H\left(f_{n}(x)\right)
$$


où $f_{E}(x)$ désigne la fonction caractéristique de l'ensemble $E$ (c'est-à-dire $f_{E}(x)=1$ sur $E$ et $=0$ sur le complémentaire de $\left.E\right)$. Le théorème I résulte alors $\mathrm{du}$ théorème 19 , et le théorème II - du théorème 20.

Remarque. Il n'y a aucune difficulté d'étendre les théorèmes I et II aux segments d'une droite (Il suffira d'observer à cet but que la circonférence est une courbe rectifiable).

Des théorèmes analogues subsistent pour le plan:

Théorème $I^{*}$. On peut attacher à toute fonction bornée $f(x, y)$ définie dans un domaine bornée, un nombre réel $H(f(x, y))$, satisfaisant aux conditions suivantes:

1) Lorsque $f_{1}(x, y)$ et $f_{2}(x, y)$ sont des fonctions bornées quelconques, définies dans le même domaine $E, c_{1}$ et $c_{2}$ deux nombres réels quelconques, on $a$ :

$$
H\left(c_{1} f_{1}(x, y)+c_{2} f_{2}(x, y)\right)=c_{1} H\left(f_{1}(x, y)\right)+c_{2} H\left(f_{2}(x, y)\right) .
$$

2) On a $H(f(x, y)) \geqslant 0$ pour $f(x, y) \geqslant 0$.

3) Lorsque $f(x, y)=c$ dans un carré $K$ aux côtes 1 , on a $H(f(x, y))=c$.

4) Lorsque $\left.f(x, y) \cong g(x, y)^{1}\right)$, nous avons $H(f(x, y))=H(g(x, y))$.

5) Lorsque $f(x, y)$ est une function intégrable $(L)$, on a

$$
H(f(x, y))=\iint_{\Sigma} f(x, y) d x d y
$$

Théorème II*. Il existe une opération $H$ satisfaisant aux conditions 1)-4) du théorème $I^{*}$ qui n'est pas identique avec l'intégrale de Lebesgue pour toutes les fonction mesurables $(L)$.

Théorème III*. A tout ensemble borné $E$ de points du plan peut être attaché un nombre $H(E)$ satisfaisant aux conditions suivantes:

1) $H(E) \geqslant 0$.

2) $H(E)=1$ lorsque $E$ est un carré de côté 1 .

3) $H\left(E_{1}+E_{2}^{\prime}\right)=H\left(E_{1}\right)+H\left(E_{2}\right)$, si $E_{1} E_{2}=0$.

4) $H\left(E_{1}\right)=H\left(E_{2}\right)$, si $E_{1} \cong E_{2}$.

5) $H(E)$ coïncide avec la mesure lebesguienne (superficielle) de l'ensemble $E$, lorsque $E$ est mesurable $(L)$.

1) Nous appelons superposables deux fonctions $f(x, y)$ et $g(x, y)$, définies dans les ensembles $E$ et $E_{1}$, lorsque $E \cong E_{1}$ et lorsqu'il existe une superposition de $E$ et $E_{1}$, telle que dans les points correspondants les fonctions $f$ et $g$ ont des valeurs égales. 
Théorème IV*. Il existe une fonction $H(E)$ satisfaisant aux conditions 1) -4) du théorème $I I I^{*}$ et un ensemble $W$ de mesure lebesguienne (superficielle) nulle, tels que $H(W)=1$.

Dém. Nous définirons comme il suit la fonction $H(f(x, y))$ du théorème $I^{*}$. Considérons un cercle de rayon $\frac{1}{2 \pi}$, ayant pour centre l'origine des coordonnées rectangulaires. $f(x, y)$ étant une fonction donnée, bornée et définie dans l'ensemble borné $E$, définissons une fonction $\varphi(\xi)$ sur la circonférence. de notre cercle de la façon suivante.

Tournons les axes $O X$ et $O Y$ d'un angle $\xi$ et désignons par $x^{\prime}, y^{\prime}$ les nouvelles coordonnées. Nous aurons donc les formules

$$
x=x^{\prime} \cos \xi-y^{\prime} \sin \xi, \quad y=x^{\prime} \sin \xi+y^{\prime} \cos \xi .
$$

Envisageons la fonction

$$
f_{1}\left(x^{\prime}, y^{\prime}\right)=f\left(x^{\prime} \cos \xi-y^{\prime} \sin \xi, x^{\prime} \sin \xi+y^{\prime} \cos \xi\right),
$$

définie dans l'ensemble $E_{1}$ des points $\left(x^{\prime}, y^{\prime}\right)$ qu'on obtient en tournant $E$ autour d'origine des coordonnées de l'angle $-\xi$.

$H\left(\varphi\left(y^{\prime}\right)\right)$ désignant l'opération du théorème 19 , définissons maintenant la fonction $g\left(x^{\prime}\right)$ d'une variable réelle $x^{\prime}$ comme il suit.

Soit $a^{\prime}$ un nombre réel donné. Si la droite $x^{\prime}=a^{\prime}$ a des points communs avec l'ensemble $E_{1}$, posons

$$
g\left(a^{\prime}\right)=H\left(f_{1}\left(a^{\prime}, y^{\prime}\right)\right)
$$

sinon, posons $g\left(a^{\prime}\right)=0$.

Posons maintenant

$$
\varphi(\xi)=H\left(g\left(x^{\prime}\right)\right)
$$

Définissons encore la fonction $\psi(x)$ pareillement comme $\varphi(\xi)$, en remplaçant seulement l'axe $O X^{\prime}$ par $O Y^{\prime}$ et inversement (et en conservant les directions des axes).

Nous définissons maintenant l'opération $H(f(x, y))$ en posant

$$
H(f(x, y))=\frac{1}{2} H[\varphi(\xi)+\psi(\xi)] .
$$

On vérifie sans peine que l'opération $H$ ainsi définie satisfait à toutes les conditions du théorème $I^{*}$. Quant au théorème III*, le raisonnement est analogue.

Pour les théorèmes $\mathrm{II}^{*}$ et $\mathrm{IV}^{*}$, je donne ici seulement une esquisse d'une démonstration. 
Considérons un corps d'hyperfonctions $\Omega\left(F^{\prime}\right)$, tel que

1) Toute hyperfonction du corps $\Omega(R)$ appartient à $\Omega\left(F^{\prime}\right)$.

2) Toute fonction $\chi(x)$ qui est égale à 1 dans un ensemble de première catégorie de M. Baire et de mesure nulle, et qui est nulle dans le complémentaire de cet ensemble, appartient à une hyperfonction $F^{\prime}$ du corps $\Omega\left(F^{\prime}\right)$.

1) et 2).

3) Le corps $\Omega(F)$ est le plus petit satisfaisant aux conditions

Définissons l'opération $A(X)$ dans le corps $\Omega(F)$, en posant

$$
A(X)=(L) \int_{0}^{1} f(x) d x
$$

où $f(x)$ est une fonction intégrable $(L)$, contenue dans $X$. Or, désignons par $P$ la même hyperfonction que dans la démonstration du théorème 20, posons $\Omega_{1}=\Omega(F, P)$ et définissons l'opération $A_{1}(X)$ dans $\Omega_{1}$, ensuite l'opération $\widehat{A}(X)$ dans le corps de toutes les hyperfonctions et enfin l'opération $H(f(x))$ d'une façon analogue comme dans la démonstration du théorème 20.

On pourrait démontrer que toute fonction $\sigma(x)$ qui est $=1$ dans un certain ensemble $E$ de mesure nulle, dont le complémentaire est de $1^{\text {re }}$ catégorie de $\mathrm{M} \mathrm{Baire,} \mathrm{et} \mathrm{qui} \mathrm{est} \mathrm{nulle} \mathrm{dans} \mathrm{le} \mathrm{com-}$ plémentaire de cet ensemble, vérifie l'inégalité

$$
H(\sigma(x))=1 .
$$

Il est en effet $\varrho(x)-\sigma(x)= \pm 1$ dans un ensemble de première catégorie et de mesure nulle, et $\varrho(x)-\sigma(x)=0$ dans le complémentaire de cet ensemble. Par conséquent l'hyperfonction $F$ contenant la fonction $\varrho(x)-\sigma(x)$ appartient à $\Omega\left(F^{\prime}\right)$, don $H(\varrho(x)-\sigma(x))=0$, ce qui donne

$$
H(\sigma(x))=1 .
$$

Ceci posé, considérons l'ensemble plan $W$ tormé des segments de longueur 1, perpendiculaires à l'axe d'abscisses dans les points de l'ensemble $E$ défini plus haut. En définissant l'opération $H$ comme dans le théorème $I^{*}$, on arrive sans peine aux théorèmes $\mathrm{II}^{*}$ et $\mathrm{IV}^{*}$. 